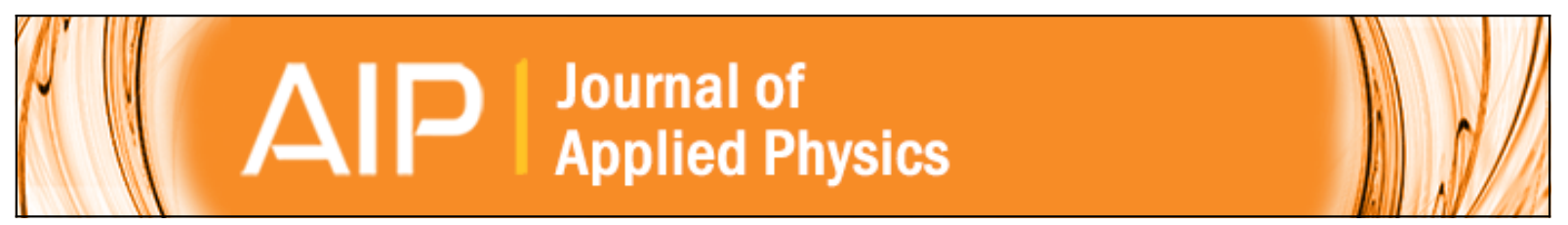

\title{
Empirical determination of the energy band gap narrowing in $p+$ silicon heavily doped with boron
}

Di Yan and Andres Cuevas

Citation: Journal of Applied Physics 116, 194505 (2014); doi: 10.1063/1.4902066

View online: http://dx.doi.org/10.1063/1.4902066

View Table of Contents: http://scitation.aip.org/content/aip/journal/jap/116/19?ver=pdfcov

Published by the AIP Publishing

\section{Articles you may be interested in}

Empirical determination of the energy band gap narrowing in highly doped $\mathrm{n}+$ silicon

J. Appl. Phys. 114, 044508 (2013); 10.1063/1.4816694

Electronic properties of titanium in boron-doped silicon analyzed by temperature-dependent photoluminescence and injection-dependent photoconductance lifetime spectroscopy

J. Appl. Phys. 104, 074510 (2008); 10.1063/1.2996252

Below bulk-band-gap photoluminescence at room temperature from heavily P-and B-doped Si nanocrystals J. Appl. Phys. 94, 1990 (2003); 10.1063/1.1590409

Femtosecond energy relaxation of nonthermal electrons injected in p-doped GaAs base of a heterojunction bipolar transistor

J. Appl. Phys. 90, 315 (2001); 10.1063/1.1372657

Boron-related minority-carrier trapping centers in p-type silicon

Appl. Phys. Lett. 75, 1571 (1999); 10.1063/1.124758

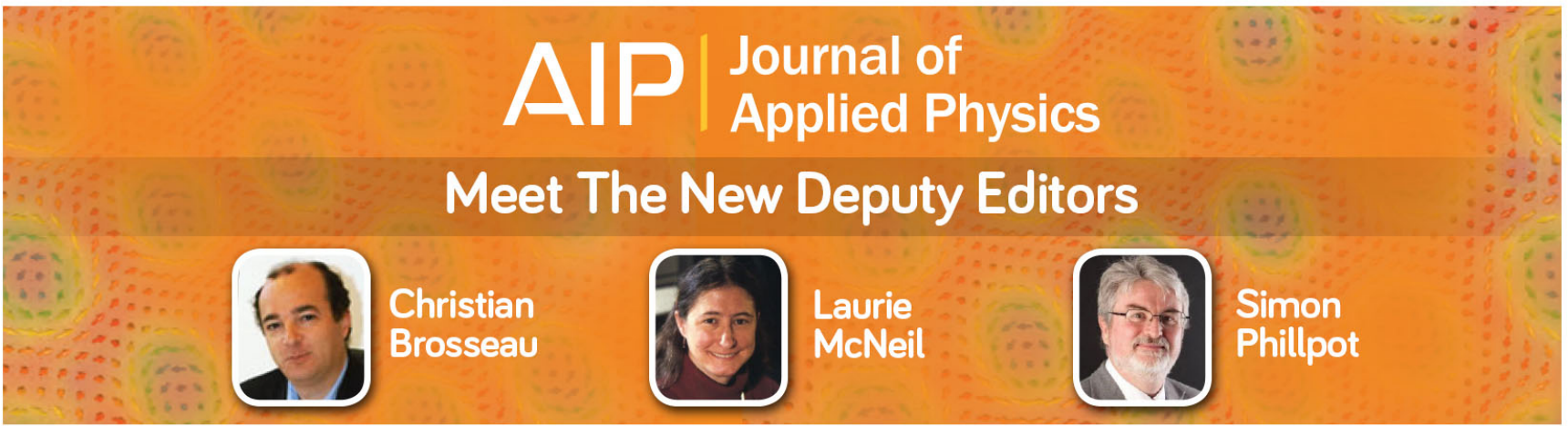




\title{
Empirical determination of the energy band gap narrowing in $\mathbf{p}^{+}$silicon heavily doped with boron
}

\author{
Di Yan ${ }^{\text {a) }}$ and Andres Cuevas \\ Research School of Engineering, The Australian National University, Canberra ACT 0200, Australia
}

(Received 1 August 2014; accepted 7 November 2014; published online 19 November 2014)

\begin{abstract}
In the analysis of highly doped silicon, energy band gap narrowing (BGN) and degeneracy effects may be accounted for separately, as a net BGN in conjunction with Fermi-Dirac statistics, or lumped together in an apparent BGN used with Boltzmann statistics. This paper presents an experimental study of silicon highly doped with boron, with the aim of evaluating the applicability of previously reported BGN models. Different boron diffusions covering a broad range of dopant densities were prepared, and their characteristic recombination current parameters $\mathrm{J}_{0}$ were measured using a contactless photoconductance technique. The BGN was subsequently extracted by matching theoretical simulations of carrier transport and recombination in each of the boron diffused regions and the measured $\mathrm{J}_{0}$ values. An evaluation of two different minority carrier mobility models indicates that their impact on the extraction of the BGN is relatively small. After considering possible uncertainties, it can be concluded that the BGN is slightly larger in $\mathrm{p}^{+}$silicon than in $\mathrm{n}^{+}$silicon, in qualitative agreement with theoretical predictions by Schenk. Nevertheless, in quantitative terms that theoretical model is found to slightly underestimate the BGN in $\mathrm{p}^{+}$silicon. With the two different parameterizations derived in this paper for the BGN in $\mathrm{p}^{+}$silicon, both statistical approaches, Boltzmann and Fermi-Dirac, provide a good agreement with the experimental data. (C) 2014 AIP Publishing LLC. [http://dx.doi.org/10.1063/1.4902066]
\end{abstract}

\section{INTRODUCTION}

The common use of highly doped regions in silicon devices, particularly in solar cells, has brought heavy-doping effects to the attention of many researchers over the years. In addition to a reduction of the minority carrier lifetime and mobility, the most important consequence of heavy doping is a change of the thermal equilibrium electron-hole product $\mathrm{p}_{0} \mathrm{n}_{0}$ compared to its low-doping value $\mathrm{n}_{\mathrm{i}}{ }^{2}$ The $\mathrm{p}_{0} \mathrm{n}_{0}$ product increases with dopant density due to energy band gap narrowing (BGN), and it decreases as a result of degeneracy effects and the subsequent need to use Fermi-Dirac statistics. A significant corpus of literature exists on experimental BGN data, for both highly doped n-type and p-type silicon. ${ }^{1}$ For many years, during the extraction of BGN values from carrier transport measurements, different minority carrier mobility and lifetime assumptions were made by different authors, causing a relatively large disagreement amongst the published results. In 1992, Klaassen et al., ${ }^{2}$ continuing the line of work started by del Alamo et al., ${ }^{1}$ reexamined the data by applying a unified mobility model for minority carriers and an updated value of the intrinsic carrier concentration in silicon, $\mathrm{n}_{\mathrm{i}}$. The, thus, updated BGN values indicated a very small difference between n-type and p-type silicon, leading to the recommendation that a single BGN parameterization could be used for both. Nevertheless, such recommendation, and common practice among many researchers, is at odds with the theoretical work of Schenk, ${ }^{3}$ which indicates that the BGN in n-type and p-type silicon should be expected to be different, slightly higher in p-type silicon.

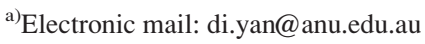

To clarify the matter, we present here a detailed study of highly doped p-type silicon, complementing a similar recent study of highly doped n-type silicon. ${ }^{4}$ The investigation is based on a broad range of boron diffused silicon samples, prepared in such a way to maximize the sensitivity to the BGN and minimize the impact of the minority carrier lifetime. The measured recombination current parameter of each diffused region $\mathrm{J}_{0}$ is compared to computer simulations with Boltzmann statistics using the, until now, widely accepted apparent $\mathrm{BGN}$ model ${ }^{5}$ (the same as for $\mathrm{n}$-type silicon). Such exercise leads to a strong discrepancy between the measured and modelled $\mathrm{J}_{0}$. The discrepancy does not disappear even if the modelling is done with Schenk's p-type BGN model ${ }^{3}$ and Fermi-Dirac statistics. Therefore, an updated BGN model for p-type silicon is required.

In Secs. II-V, we present details of sample preparation and experimental characterization. The impact of the minority carrier parameters, mobility and Auger recombination, on the extraction of the BGN from the experimental measurements is then quantified. Finally, simple empirical models are derived to calculate either the apparent BGN (i.e., encompassing the effects of degeneracy) or the net BGN as a function of the dopant density.

\section{EXPERIMENTAL METHODS}

A diversity of dopant density profiles were produced by thermal boron diffusion, followed by oxidation/drive-in steps, on $100 \Omega$-cm high-resistivity n-type silicon wafers $\left(\mathrm{N}_{\mathrm{D}}=4.43 \times 10^{13} \mathrm{~cm}^{-3}\right)$ with a thickness of $400 \pm 20 \mu \mathrm{m}$. The boron pre-deposition step was performed within a temperature range of $900-1000^{\circ} \mathrm{C}$ using $\mathrm{BBr}_{3}$ as a dopant 
source. The drive-in step was carried out in pure oxygen at a temperature of $1000^{\circ} \mathrm{C}$ for $10-100 \mathrm{~min}$, after having removed the boron-silica glass. As a result, a total of 35 different dopant profiles, with sheet resistances between $19.6 \pm 0.2 \Omega / \mathrm{sq}$ and $206.6 \pm 22 \Omega / \mathrm{sq}$ and surface dopant concentrations from $(6.14 \pm 0.01) \times 10^{18} \mathrm{~cm}^{-3}$ to $(1.31 \pm 0.02)$ $\times 10^{20} \mathrm{~cm}^{-3}$, were generated. The corresponding dopant density profiles, some of which are shown in Fig. 1, were measured using an electrochemical capacitance-voltage instrument (WEP Wafer Profile CVP21). This method gives the total concentration of substitutional, electrically active dopant atoms, which become ionized during the measurement. Incomplete ionization at room temperature, ${ }^{6}$ whereas possible, is very small for the high dopant densities explored here; nevertheless, we have included incomplete ionization in the theoretical modelling. The surface dopant concentration was determined by matching the sheet resistance calculated from an integration of the dopant profile to the value measured with both a four point probe (Signatone, model S-301-4) and a calibrated inductive-coil conductance tester (Sinton Instruments, WCT 120).

After the thermal steps, all silicon oxides were removed from the surface in dilute $\mathrm{HF}$ and the wafers were cleaned in standard solutions based on ammonium hydroxide, hydrochloric acid, and hydrogen peroxide (RCA clean), plus another HF dip. Semi-transparent aluminum layers with a thickness of $10-20 \mathrm{~nm}$ were deposited on both sides of the wafers. For each metal-coated sample, that is, for every dopant profile, the recombination current density parameter $\mathrm{J}_{0}$ was measured at room temperature by both transient photoconductance decay (PCD) and QuasiSteady-State photoconductance (QSSPC), following the method described in Ref. 7, that is, by a linear fit of the inverse effective lifetime, corrected by Auger recombination in the highly injected silicon wafer, ${ }^{8}$ at an excess carrier density in the range of $\Delta \mathrm{n}=0.5-1 \times 10^{15} \mathrm{~cm}^{-3}$. This simple method assumes that $\Delta \mathrm{n}$ is approximately constant over the wafer thickness. We re-analyzed the measurements

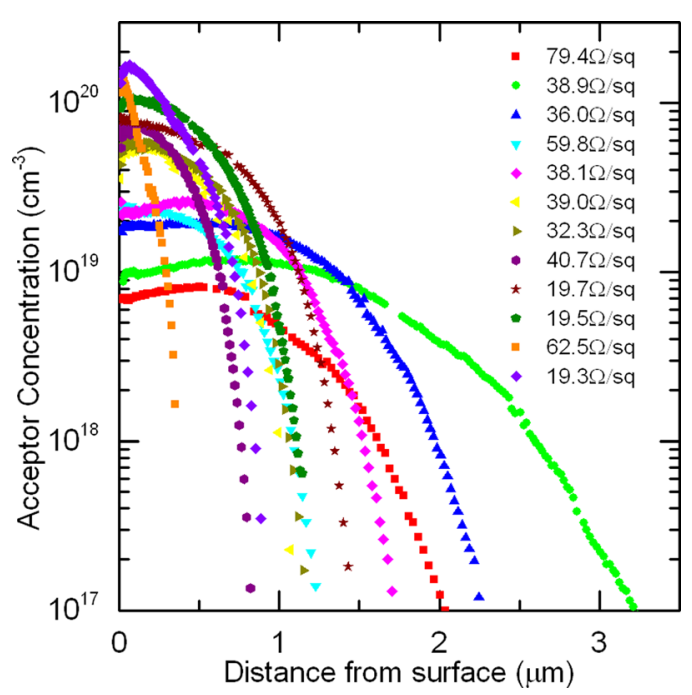

FIG. 1. Electrically active boron concentration profiles of several silicon samples with different sheet resistances and acceptor concentrations. using the numerical analysis procedure suggested in Ref. 9, which takes into account possible non-uniformities in excess carrier distribution. Even in the most extreme cases (highest $\mathrm{J}_{0}$ ), we found that the maximum relative difference compared to the simple analysis is less than $10 \%$, that is, smaller than the estimated overall experimental error of $\pm 10 \%$. The $\mathrm{J}_{0}$ values at $300 \mathrm{~K}$ are plotted as a function of the sheet resistance of the diffusion in Fig. 2.

\section{PREDICTION OF THE RECOMBINATION PARAMETER $\mathrm{J}_{0}$ BY COMPUTER SIMULATION}

It is straightforward to calculate the recombination current parameter $\mathrm{J}_{0}$, which corresponds to a given dopant profile. A simple and flexible analytical minority-carrier transport model ${ }^{10}$ has been used in this work, after confirming that it provides good accuracy in comparison to numerical simulations. ${ }^{11}$ The physical material parameters required for the simulations are the minority carrier lifetime $\tau_{\mathrm{n}}$, the minority carrier mobility $\mu_{\mathrm{n}}$, the surface recombination velocity $S_{n}$, and the equilibrium $p_{0} n_{0}$ product. Since the surface is metalized, we have assumed that carriers reaching it do so at their thermal velocity, that is, the surface recombination velocity for electrons is $S_{n}=3 \times 10^{6} \mathrm{~cm} / \mathrm{s}^{5}$ considering the one-dimensional restriction imposed by the surface plane on the random thermal motion of carriers (approximately a factor $1 / 3$ ). We made the reasonable assumption that Auger recombination is dominant in heavily doped silicon, and that Shockley-Read Hall recombination is negligible. Therefore, we have computed $\tau_{\mathrm{n}}$ as a function of dopant density using the empirical model proposed by Richter et al. ${ }^{8}$ The minority electron mobility $\mu_{\mathrm{n}}$ has been calculated with Klaassen's unified mobility model. ${ }^{12}$ The uncertainty related to these assumptions is discussed below.

At high dopant concentrations, the thermal equilibrium $\mathrm{p}_{0} \mathrm{n}_{0}$ product changes with respect to its normal value $n_{i}^{2}$ (the intrinsic carrier density in lowly doped silicon is

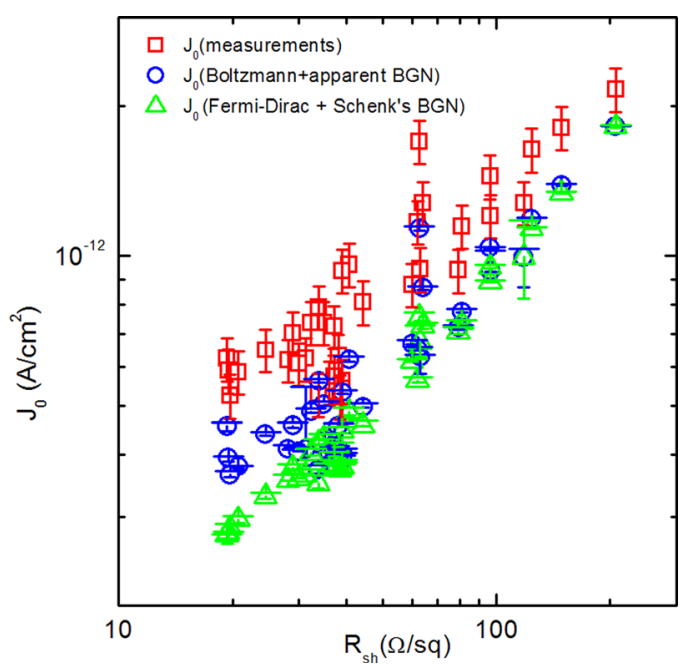

FIG. 2. Measured recombination current parameter $\mathrm{J}_{0}$ as a function of the sheet resistance of the boron diffusions, compared to computer simulations based on Fermi-Dirac statistics with Schenk's BGN, or Boltzmann statistics with the same apparent BGN as that of n-type silicon. 
$\mathrm{n}_{\mathrm{i}}=9.65 \times 10^{9} \mathrm{~cm}^{-3}$ at $300 \mathrm{~K}$ (Ref. 13)) due to energy bandgap narrowing $\Delta E_{g}$ and to Fermi-Dirac statistics. An effective intrinsic carrier concentration $n_{\text {ieff }}$ can be determined as

$$
p_{0} \cdot n_{0}=n_{\text {ieff }}^{2}=n_{i}^{2} \frac{\frac{n_{o}}{N_{c}}}{\exp \left(F_{1 / 2}^{-1}\left(\frac{n_{o}}{N c}\right)\right)} \exp \left(\frac{\Delta E_{g}}{K T}\right) .
$$

Alternatively, the use of Boltzmann statistics may be forced, leading to an apparent $\mathrm{BGN} \Delta E_{g}^{a p p}$ defined as

$$
p_{0} \cdot n_{0}=n_{\text {ieff }}^{2}=n_{i}^{2} \exp \left(\frac{\Delta E_{g}^{a p p}}{K T}\right) .
$$

We have performed the simulations in two different scenarios. The first following Eq. (1), with Schenk's theoretical BGN model ${ }^{3}$ for p-type silicon together with Fermi-Dirac statistics. The second, quite extended in the research community, using an apparent BGN parameterization identical to that reported for n-type silicon, ${ }^{5}$ together with Boltzmann statistics, as per Eq. (2). The resulting values of the $\mathrm{p}_{0} \mathrm{n}_{0}$ product are plotted in Fig. 3 as a function of the dopant concentration in p-type silicon, assumed to be equal to the majority carrier (hole) concentration. It can be observed that BGN effects tend to increase the $\mathrm{p}_{0} \mathrm{n}_{0}$ product, hence the minority carrier concentration. But once the dopant concentration surpasses the effective density of states in the valence band $\mathrm{N}_{\mathrm{v}}$, which has a value close to $3 \times 10^{19} \mathrm{~cm}^{-3}$, degeneracy effects start reducing the $\mathrm{p}_{0} \mathrm{n}_{0}$ product. In other words, degeneracy effects partially compensate for the consequences of BGN. Fig. 3 shows that the two calculations of the $\mathrm{p}_{0} \mathrm{n}_{0}$ product are almost identical over a broad range of dopant densities, but they diverge at very high dopant concentrations. This indicates that the parameterization used here for

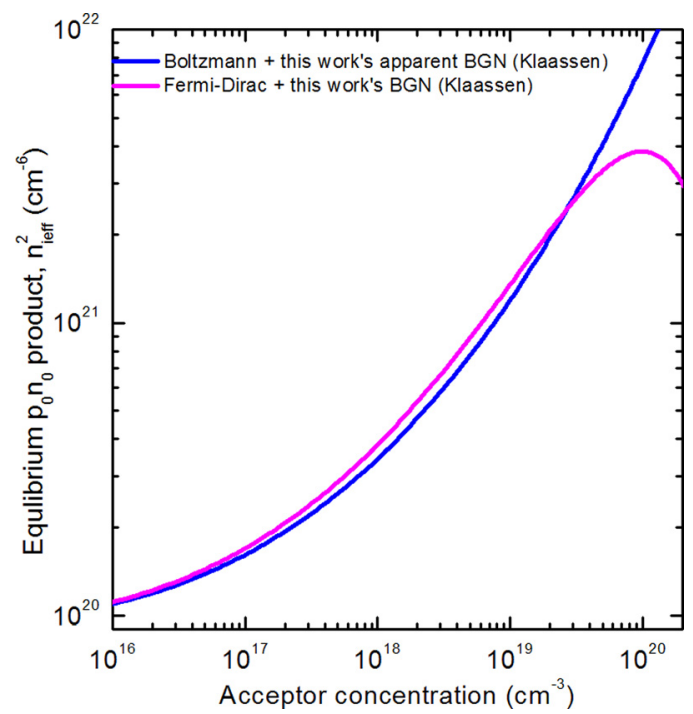

FIG. 3. Equilibrium $p_{0} n_{0}$ product in highly doped $p^{+}$silicon, computed with either Boltzmann or Fermi-Dirac statistics with the corresponding empirical expressions for the energy band gap narrowing derived in this paper and Klaassen's minority carrier mobility.
$\Delta E_{g}^{a p p}$ does not perfectly account for degeneracy effects for dopant densities in the vicinity of $1 \times 10^{20} \mathrm{~cm}^{-3}$ and above.

A comparison between the simulated and experimental $\mathrm{J}_{0}$ values for all the samples fabricated for this study is presented in Fig. 2. As can be observed, the n-type apparent BGN model ${ }^{5}$ and Boltzmann statistics cannot reproduce the measurements of $\mathrm{J}_{0}$ accurately. The second scenario based on Schenk's BGN model and Fermi-Dirac statistics leads to an even stronger disagreement with the experimental $\mathrm{J}_{0} \mathrm{val}-$ ues. The discrepancies are greater for the diffusions with the lowest sheet resistances, that is, with the highest dopant concentrations. Since both simulation scenarios underestimate the recombination current density $\mathrm{J}_{0}$, the experiments indicate that the BGN in highly doped $\mathrm{p}^{+}$silicon is likely higher than previously thought.

\section{EXTRACTION OF THE ENERGY BANDGAP NARROWING IN $\mathrm{P}^{+}$SILICON}

The difference between experimental and simulated $\mathrm{J}_{0}$ values indicates that a reevaluation of the BGN in heavily doped p-type silicon is necessary. The objective of this section is to derive an empirical BGN model as a function of dopant density that provides consistency between the simulated and the measured $\mathrm{J}_{0}$ values. As a starting point, we assume a simple expression for the BGN as a function of dopant concentration

$$
\Delta \mathrm{E}_{\mathrm{g}}\left(\mathrm{N}_{\mathrm{A}}\right)=\mathrm{A}\left[\ln \left(\frac{\mathrm{N}_{\mathrm{A}}}{\mathrm{N}_{\mathrm{ref}}}\right)\right]^{\mathrm{b}}+\mathrm{C},
$$

where $\mathrm{A}, \mathrm{b}$, and $\mathrm{C}$ are constants and $\mathrm{N}_{\text {ref }}$ represents a reference dopant concentration where BGN effects are still negligible. This expression provides a good fit to Schenk's theoretical $\mathrm{BGN}$ model when $\mathrm{C}=0, \mathrm{~b}=3$, $\mathrm{N}_{\text {ref }}=1 \times 10^{14} \mathrm{~cm}^{-3}$, and $\mathrm{A}=4.20 \times 10^{-5} \mathrm{eV}$. Based on that we fix three of the constants in Eq. (3) to $\mathrm{C}=0, \mathrm{~b}=3$, and $\mathrm{N}_{\mathrm{ref}}=1 \times 10^{14} \mathrm{~cm}^{-3}$, and then we iteratively perform simulations for each dopant profile until a value of the constant $\mathrm{A}$ is found by matching the simulated $\mathrm{J}_{0}$ and the measured $\mathrm{J}_{0}$. The results of such procedure for the 35 different boron diffusions are given in Table III of the Appendix. Despite the diversity of dopant profiles in the study, they all lead to quite similar values of the constant $\mathrm{A}$; averaging all of them we determine a global value of the parameter A which approximately represents BGN effects in all of them collectively. By repeating the analysis twice, once with Boltzmann statistics and a second time using Fermi-Dirac statistics, we obtain two different values of the constant $\mathrm{A}$, the first corresponding to the apparent $\mathrm{BGN}\left(\mathrm{A}=4.32 \times 10^{-5} \mathrm{eV}\right)$, and the second to the net $\mathrm{BGN}\left(\mathrm{A}=4.72 \times 10^{-5} \mathrm{eV}\right)$. Alternatively, some of the most commonly used apparent $\mathrm{BGN}$ models ${ }^{5,14,15}$ are based on a simpler expression with $\mathrm{b}=1$ and $\mathrm{C}=0$; in this case, a value of $\mathrm{N}_{\text {ref }}=1 \times 10^{17} \mathrm{~cm}^{-3}$ gives the best fit to our experimental data, with a resulting constant $\mathrm{A}=1543$ $\times 10^{-5} \mathrm{eV}$. The values of the constants associated with each of the different BGN models are summarized in Table II. These calculations are based on an intrinsic carrier concentration of $n_{i}=9.65 \times 10^{9} \mathrm{~cm}^{-3}$ at a temperature of $300 \mathrm{~K}$. 
TABLE I. Measured recombination current density $\mathrm{J}_{0}$ of two boron profiles with similar surface concentrations but different sheet resistances. The surface was metalized with aluminium.

\begin{tabular}{lccc}
\hline \hline & $\mathrm{N}_{\text {surf }}\left(\mathrm{cm}^{-3}\right)$ & $\mathrm{R}_{\text {sh }}(\Omega / \mathrm{sq})$ & $\mathrm{J}_{0}\left(\mathrm{~A} / \mathrm{cm}^{3}\right)$ \\
\hline Sample 1 & $3.39 \times 10^{19}$ & 35 & $7.36 \times 10^{-13}$ \\
Sample 2 & $3.44 \times 10^{19}$ & 62 & $1.17 \times 10^{-12}$ \\
\hline
\end{tabular}

We have evaluated the possible influence of incomplete ionization and confirmed that the difference in the constant $\mathrm{A}$ that results from including or not an incomplete ionization model ${ }^{16,17}$ is within the error range of the results, as given in Table II. Therefore, the impact of incomplete ionization is negligible in this work.

\section{A. Uncertainties in BGN extraction due to carrier mobility and lifetime}

In addition to measurement error, indicated by the error bars in Fig. 2, the extraction of the BGN via modeling may be affected by the remaining carrier transport and recombination parameters. In particular, the minority carrier lifetime may be affected by the presence of defects or dopant precipitates, or simply by the uncertainties in the Auger recombination parameters. To minimize its impact, we have designed the experiment so that the total recombination in the diffused region happens mostly at the surface, rather than in the volume. We ensured a very high surface recombination velocity by directly depositing aluminum onto the silicon. This means that carrier recombination is limited by the supply of carriers towards the surface, that is, by the minority diffusivity (or mobility). An approximate, first order solution for such transport-limited recombination regime is ${ }^{10}$

$$
\mathrm{J}_{\mathrm{op}^{+}}=\left(\int_{0}^{\mathrm{W}_{\mathrm{p}^{+}}} \frac{1}{\mathrm{qn}_{0} \mathrm{D}_{\mathrm{n}}} \mathrm{dx}\right)^{-1},
$$

where $\mathrm{q}$ is the elementary charge, $\mathrm{W}_{\mathrm{p}+}$ is the width of the diffused region, $\mathrm{n}_{0}$ is the equilibrium electron density (within which the BGN is embedded), and $\mathrm{D}_{\mathrm{n}}$ is the minority carrier diffusion coefficient. As Eq. (4) indicates a high carrier mobility or a short distance to be covered by the carriers in their trip to the surface will result in a large recombination parameter $\mathrm{J}_{0}$, and vice versa. This can be illustrated by comparing two dopant profiles with similar surface dopant concentration and different diffusion depth, as those in Table I. Transport to the surface occurs more readily for the thinner diffusion, especially when it is shorter than the diffusion length of minority carriers, leading to a higher $\mathrm{J}_{0}$.

Generally, Auger recombination in the subsurface region represents only a small amount of the total recombination in the transport limited case, and this drastically reduces the impact of Auger recombination model in the simulation of these samples. To confirm this, we applied different Auger recombination models by Dziewior and Schmid, ${ }^{18}$ Kerr and Cuevas, ${ }^{19}$ Altermatt et al., ${ }^{20}$ and Richter
TABLE II. Characteristic parameter A corresponding to the two empirical BGN models, net $\Delta \mathrm{E}_{\mathrm{g}}$ and apparent $\Delta \mathrm{E}_{\mathrm{g}}$ app , derived in this paper using the minority carrier mobility of either Klaassen et al. or Swirhun et al. The BGN parameters for $\mathrm{n}^{+}$silicon derived in Ref. 4 are included for comparison. For the calculation with the third order function Eq. (1), values of $\mathrm{C}=0$ and $\mathrm{N}_{\text {ref }}=1 \times 10^{14} \mathrm{~cm}^{-3}$ were used. For the apparent BGN model with $\mathrm{b}=1$, last two rows in the table, constant values of $\mathrm{C}=0$ and $\mathrm{N}_{\mathrm{ref}}=1 \times 10^{17} \mathrm{~cm}^{-3}$ were used.

\begin{tabular}{lccccc}
\hline \hline & Dopant type & Statistics & Mobility model & $\mathrm{A}\left(\times 10^{-5} \mathrm{eV}\right)$ & $\mathrm{b}$ \\
\hline$\Delta \mathrm{E}_{\mathrm{g}}{ }^{\text {app }}$ & $\mathrm{N}^{+}$ & Boltzmann & Klaassen & $(3.67 \pm 0.20)$ & 3 \\
$\Delta \mathrm{E}_{\mathrm{g}}$ & $\mathrm{N}^{+}$ & Fermi-Dirac & Klaassen & $(4.20 \pm 0.30)$ & 3 \\
$\Delta \mathrm{E}_{\mathrm{g}}{ }^{\text {app }}$ & $\mathrm{p}^{+}$ & Boltzmann & Klaassen & $(4.32 \pm 0.12)$ & 3 \\
$\Delta \mathrm{E}_{\mathrm{g}}$ app & $\mathrm{p}^{+}$ & Boltzmann & Swirhun & $(4.12 \pm 0.15)$ & 3 \\
$\Delta \mathrm{E}_{\mathrm{g}}$ & $\mathrm{p}^{+}$ & Fermi-Dirac & Klaassen & $(4.72 \pm 0.12)$ & 3 \\
$\Delta \mathrm{E}_{\mathrm{g}}$ & $\mathrm{p}^{+}$ & Fermi-Dirac & Swirhun & $(4.53 \pm 0.16)$ & 3 \\
$\Delta \mathrm{E}_{\mathrm{g}}$ app & $\mathrm{p}^{+}$ & Boltzmann & Klaassen & $(1543 \pm 46)$ & 1 \\
$\Delta \mathrm{E}_{\mathrm{g}}$ & $\mathrm{p}^{+}$ & Boltzmann & Swirhun & $(1476 \pm 48)$ & 1 \\
\hline \hline
\end{tabular}

et $a l .{ }^{8}$ to the analysis, and found that the resulting $\mathrm{J}_{0}$ values are very similar, changing by less than $1 \%$ for all the samples.

On the other hand, Eq. (4) indicates that the extraction of the BGN (via $\mathrm{n}_{0}$ ) is affected by the assumed carrier mobility. We have investigated the impact on the simulations of two different mobility parameterizations, proposed by Klaassen et al. ${ }^{12}$ and by Swirhun et al., ${ }^{21}$ respectively. These two empirical mobility models, together with relevant experimental data, are illustrated in Fig. 4. Although the difference between both mobility models is less than the scatter in experimental data, Klaassen's model gives a slightly lower minority electron mobility in highly doped p-type silicon $\left(>2 \times 10^{17} \mathrm{~cm}^{-3}\right)$. As shown by Eq. (4), lower mobility values result in lower recombination parameters $\mathrm{J}_{0}$, hence, to achieve a given $\mathrm{J}_{0}$ with a lower mobility a higher BGN will be needed. Therefore, Klaassen's mobility model can be

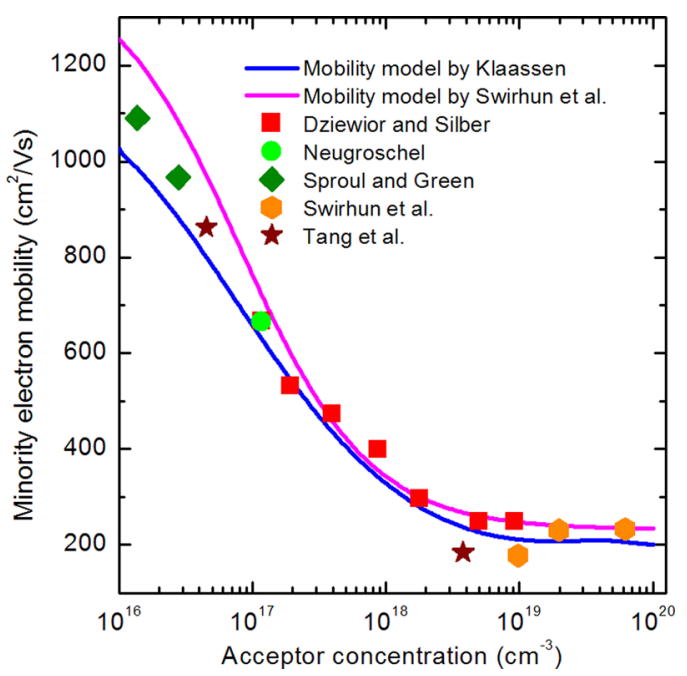

FIG. 4. Minority electron mobility as a function of dopant concentration calculated with Klaassen (blue line) and Swirhun et al. (pink line) parameterizations. Experimental measurements by Dziewior and Sliber, ${ }^{22}$ Neugroschel, ${ }^{23}$ Sproul et al., ${ }^{24}$ Swirhun et al., ${ }^{21}$ and Tang et al. ${ }^{25}$ are represented as symbols. 
expected to result in a slightly higher BGN than Swirhun's model in the analysis of our experiments.

We have repeated the analysis of all the 35 samples using either Klaassen's mobility model or the mobility model of Swirhun et al., together with the Auger recombination model of Ref. 8, noting that the specific bulk recombination model plays only a minor role in the samples analyzed here. A surface recombination velocity of $3 \times 10^{6} \mathrm{~cm} / \mathrm{s}$ is used as the boundary condition at the metal coated surface. The results of the analysis, that is, the values of the coefficient A corresponding to each of the two mobility models and to either the apparent $\mathrm{BGN}$ or net $\mathrm{BGN}$ are summarized in Table II. As expected, the coefficient A that results from using Klaassen's mobility model is larger than that from Swirhun's model, but the main point is that the difference between them is relatively small; in fact, the corresponding error margins overlap.

The BGN parameters for n-type silicon, determined in Ref. 4, are also shown in Table II. Irrespective of which mobility model is assumed, either Klaassen's or Swirhun's, the BGN extracted from the p-type boron diffused samples is larger than that for n-type phosphorus diffused regions, which is qualitatively consistent with theoretical studies. ${ }^{3}$

\section{B. Comparison between BGN models}

The four variants of the empirical BGN expression for boron doped silicon given in Table II are graphically compared in Fig. 5 to Schenk's theoretical BGN model (yellow continuous line) as well as to previously published experimental data for $\mathrm{p}+$ silicon. The net and apparent BGN parameterizations derived from the mobility model of Swirhun et al. are shown as continuous lines (red for FermiDirac and green for Boltzmann). The curve for the apparent $\Delta \mathrm{E}_{\mathrm{g}}{ }^{\text {app }}$ extracted with Swirhun's mobility model falls on top of Schenk's net $\Delta \mathrm{E}_{\mathrm{g}}$, which of course is merely coincidental, since the latter is based on Fermi-Dirac statistics and the former is not. The relevant comparison is between Schenk's model and the net $\Delta \mathrm{E}_{\mathrm{g}}$ derived in this paper (red continuous line); the experimental results from this study indicate that the net $\Delta \mathrm{E}_{\mathrm{g}}$ in boron doped silicon is higher than predicted by Schenk's theoretical model (by about $10 \mathrm{meV}$ at $1 \times 10^{20} \mathrm{~cm}^{-3}$ ). It should be noted that the experiments in this paper support the BGN empirical models only up to a dopant density of $1.3 \times 10^{20} \mathrm{~cm}^{-3}$.

The discontinuous lines represent both the net and apparent BGN models obtained from Klaassen's mobility model (red for Fermi-Dirac and blue for Boltzmann). For a given statistical model, the BGN values extracted with Klaassen's mobility are always higher than those derived with Swirhun's mobility. The four BGN expressions are in reasonably good agreement with previously reported experimental data. In fact, the differences between the BGN expressions due to different statistics or to different mobility models are smaller than the scatter in those experimental data. Over the full range of dopant concentrations explored here, the net $\mathrm{BGN} \Delta \mathrm{E}_{\mathrm{g}}$ is higher than the apparent BGN $\Delta \mathrm{E}_{\mathrm{g}}^{\text {app }}$, the discrepancy between them becoming more pronounced at the higher dopant concentrations. A good correlation can be observed between the net $\Delta \mathrm{E}_{\mathrm{g}}$ derived with the
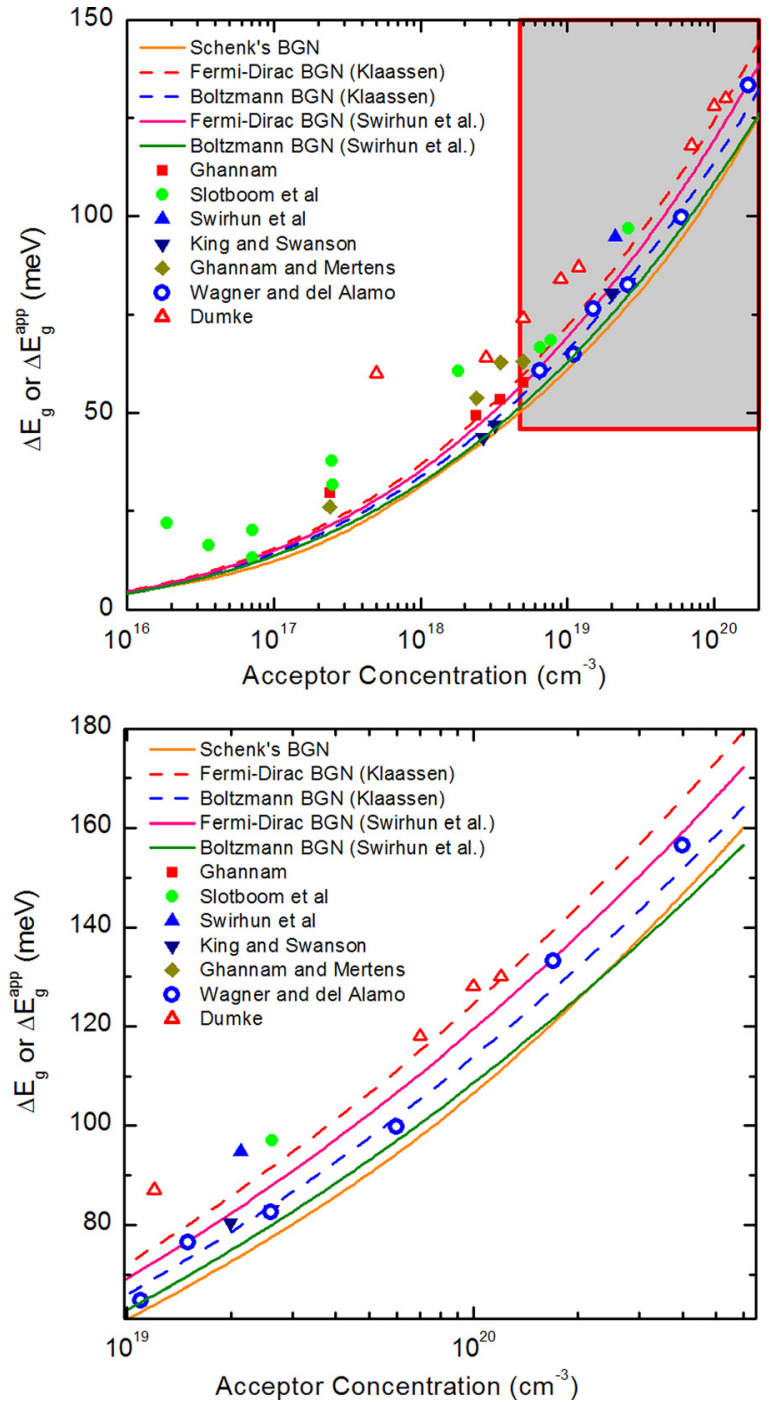

FIG. 5. Energy bandgap narrowing as a function of dopant concentration in heavily doped p-type silicon. The dashed lines represent BGN models derived by using mobility of Swrihun et al. The continuous lines represent BGN models obtained from Klaassen's mobility, as well as Schenk's theoretical p-type BGN model. Electronic measurements by Slotboom and de Graaff, 28 Swirhun et al., ${ }^{21}$ King and Swanson, ${ }^{29}$ and Ghannam and Mertens. ${ }^{27}$ They were re-calculated with Klaassen's mobility model and $\mathrm{n}_{\mathrm{i}}=9.65 \times 10^{9} \mathrm{~cm}^{-3}$. Photoluminescence measurements from Wagner and del Alamo ${ }^{26}$ and Dumke. ${ }^{30}$ The bottom shows the enlarged figure of the shadow region of top figure. It is in a dopant range from $10^{19} \mathrm{~cm}^{-3}$ to $6 \times 10^{20} \mathrm{~cm}^{-3}$.

mobility model of Swirhun et al. and the photoluminescence measurements of the BGN by Wagner and del Alamo; ${ }^{26}$ such agreement is significant, since photoluminescence measurements also give the net $\Delta \mathrm{E}_{\mathrm{g}}$. Based on this agreement, it appears that the mobility expression proposed by Swirhun $e t$ al. is more appropriate for highly doped p-type silicon than the more widely used model of Klaassen, although the difference between the resulting expressions for the BGN is relatively small.

\section{CONCLUSION}

In this paper, updated BGN models for boron doped $\mathrm{p}^{+}$ silicon have been derived from a combination of experimental measurements and computer simulations. The wellestablished approach to model minority carrier transport and 
recombination in highly doped p-type silicon, based on Fermi-Dirac statistics and Schenk's theoretical BGN, underestimates the recombination parameter $\mathrm{J}_{0}$ for all the boron diffusions prepared for this study; the level of underestimation being up to a factor of 2.3 for the most heavily doped diffusions. A second approach, also quite extended among the scientific community, has been based on assuming that the apparent $\mathrm{BGN}$ in $\mathrm{p}^{+}$silicon is approximately the same as in $\mathrm{n}^{+}$silicon, together with Boltzmann statistics. Again, we have found that such approach underestimates $\mathrm{J}_{0}$, up to a factor of 1.8 in some samples. Our measurements strongly indicate that the $\mathrm{BGN}$ in $\mathrm{p}^{+}$silicon is higher than in $\mathrm{n}^{+}$silicon.

To achieve a good agreement between modelled and measured recombination parameter $\mathrm{J}_{0}$, it is necessary to use the empirical expression for the BGN derived in this paper. Two of them, one for the apparent BGN and another for the net BGN, have been derived. They are approximately equivalent to each other, as long as they are used with the appropriate carrier statistics, either Boltzmann or FermiDirac. These expressions are the result of an averaging procedure over 35 different boron diffused samples, which are representative of most boron diffused regions used in silicon solar cell technology. Nevertheless, the highest dopant density explored in this paper is $1.3 \times 10^{20} \mathrm{~cm}^{-3}$, and in each of those samples the dopant concentration varies with position, which means that the $\Delta \mathrm{E}_{\mathrm{g}}$ extracted from each of them is the result of a weighted average of BGN effects over the full thickness of the diffused region. Despite those limitations, the proposed empirical expressions are appropriate to model the recombination current of a broad range of boron doped regions.

\section{APPENDIX: SUMMARY OF THE BORON DIFFUSED SAMPLES}

TABLE III. Summary of the boron diffusions used in this work, including sheet resistance and surface concentration. The $\mathrm{J}_{0}$ measurements of the aluminium coated diffusions are shown, and revaluated for $\mathrm{n}_{\mathrm{i}}=9.65 \times 10^{9} \mathrm{~cm}^{-3}$. The corresponding coefficients A calculated by using either Boltzmann or Fermi-Dirac statistics in different mobility models (Klaassen and Swirhun) are also shown. Constants, $\mathrm{b}=3, \mathrm{~N}_{\text {ref }}=10^{14} \mathrm{~cm}^{-3}$, and $\mathrm{C}=0$, are used for these calculations.

\begin{tabular}{|c|c|c|c|c|c|c|c|c|}
\hline Sample & $\begin{array}{c}\mathrm{Ns} \\
\left(\mathrm{cm}^{-3}\right)\end{array}$ & $\begin{array}{l}\text { Diffusion } \\
\text { depth } \\
(\mu \mathrm{m})\end{array}$ & $\begin{array}{c}\mathrm{R}_{\mathrm{sh}} \\
(\Omega / \mathrm{sq})\end{array}$ & $\begin{array}{c}\mathrm{J}_{0}(\text { measured }) \\
\left(\mathrm{A} / \mathrm{cm}^{2}\right)\end{array}$ & $\begin{array}{c}\text { A (Fermi-Dirac } \\
+ \text { Klaassen })\left(\times 10^{-5} \mathrm{eV}\right)\end{array}$ & $\begin{array}{c}\text { A (Boltzmann } \\
+ \text { Klaassen })\left(\times 10^{-5} \mathrm{eV}\right)\end{array}$ & $\begin{array}{c}\text { A (Fermi-Dirac } \\
+ \text { Swirhun })\left(\times 10^{-5} \mathrm{eV}\right)\end{array}$ & $\begin{array}{c}\text { A (Boltzmann } \\
+ \text { Swirhun })\left(\times 10^{-5} \mathrm{eV}\right)\end{array}$ \\
\hline 1 & $6.14 \times 10^{18}$ & 1.02 & 206.65 & $2.16 \times 10^{-12}$ & 4.38 & 4.27 & 4.13 & 4.02 \\
\hline 2 & $6.91 \times 10^{18}$ & 2.10 & 79.41 & $9.38 \times 10^{-13}$ & 4.58 & 4.44 & 4.32 & 4.18 \\
\hline 3 & $8.71 \times 10^{18}$ & 3.74 & 38.98 & $5.12 \times 10^{-13}$ & 4.61 & 4.43 & 4.35 & 4.18 \\
\hline 4 & $9.32 \times 10^{18}$ & 3.27 & 38.55 & $5.23 \times 10^{-13}$ & 4.65 & 4.48 & 4.39 & 4.23 \\
\hline 5 & $1.04 \times 10^{19}$ & 0.95 & 148.74 & $1.81 \times 10^{-12}$ & 4.56 & 4.40 & 4.31 & 4.15 \\
\hline 6 & $1.11 \times 10^{19}$ & 1.24 & 96.34 & $1.20 \times 10^{-12}$ & 4.53 & 4.34 & 4.28 & 4.09 \\
\hline 7 & $1.24 \times 10^{19}$ & 1.05 & 124.10 & $1.63 \times 10^{-12}$ & 4.65 & 4.47 & 4.41 & 4.22 \\
\hline 8 & $1.36 \times 10^{19}$ & 2.53 & 39.04 & $5.62 \times 10^{-13}$ & 4.67 & 4.45 & 4.43 & 4.21 \\
\hline 9 & $1.50 \times 10^{19}$ & 1.41 & 81.11 & $1.15 \times 10^{-12}$ & 4.77 & 4.57 & 4.53 & 4.33 \\
\hline 10 & $1.67 \times 10^{19}$ & 1.41 & 62.88 & $9.42 \times 10^{-13}$ & 4.64 & 4.39 & 4.42 & 4.17 \\
\hline 11 & $1.69 \times 10^{19}$ & 2.52 & 37.11 & $5.74 \times 10^{-13}$ & 4.66 & 4.41 & 4.44 & 4.19 \\
\hline 12 & $1.71 \times 10^{19}$ & 2.53 & 36.02 & $5.59 \times 10^{-13}$ & 4.66 & 4.40 & 4.44 & 4.18 \\
\hline 13 & $1.82 \times 10^{19}$ & 2.95 & 33.74 & $5.29 \times 10^{-13}$ & 4.70 & 4.45 & 4.48 & 4.23 \\
\hline 14 & $2.13 \times 10^{19}$ & 0.77 & 96.10 & $1.44 \times 10^{-12}$ & 4.62 & 4.32 & 4.42 & 4.11 \\
\hline 15 & $2.27 \times 10^{19}$ & 1.27 & 59.78 & $8.79 \times 10^{-13}$ & 4.55 & 4.25 & 4.34 & 4.05 \\
\hline 16 & $2.32 \times 10^{19}$ & 1.59 & 44.31 & $8.09 \times 10^{-13}$ & 4.87 & 4.59 & 4.66 & 4.38 \\
\hline 17 & $2.46 \times 10^{19}$ & 1.78 & 37.13 & $7.23 \times 10^{-13}$ & 4.90 & 4.60 & 4.70 & 4.39 \\
\hline 18 & $2.61 \times 10^{19}$ & 1.80 & 38.13 & $6.32 \times 10^{-13}$ & 4.63 & 4.31 & 4.43 & 4.11 \\
\hline 19 & $3.01 \times 10^{19}$ & 1.79 & 29.91 & $6.09 \times 10^{-13}$ & 4.73 & 4.33 & 4.56 & 4.16 \\
\hline 20 & $3.39 \times 10^{19}$ & 1.23 & 34.87 & $7.36 \times 10^{-13}$ & 4.69 & 4.23 & 4.53 & 4.07 \\
\hline 21 & $3.44 \times 10^{19}$ & 1.01 & 61.99 & $1.17 \times 10^{-12}$ & 4.97 & 4.52 & 4.80 & 4.36 \\
\hline 22 & $3.55 \times 10^{19}$ & 1.07 & 39.01 & $9.37 \times 10^{-13}$ & 4.94 & 4.44 & 4.79 & 4.28 \\
\hline 23 & $3.56 \times 10^{19}$ & 1.73 & 31.29 & $6.23 \times 10^{-13}$ & 4.75 & 4.36 & 4.57 & 4.18 \\
\hline 24 & $3.70 \times 10^{19}$ & 1.58 & 28.05 & $6.19 \times 10^{-13}$ & 4.74 & 4.29 & 4.58 & 4.13 \\
\hline 25 & $4.30 \times 10^{19}$ & 1.24 & 32.31 & $7.37 \times 10^{-13}$ & 4.75 & 4.22 & 4.60 & 4.06 \\
\hline 26 & $4.82 \times 10^{19}$ & 1.22 & 28.85 & $7.03 \times 10^{-13}$ & 4.77 & 4.23 & 4.63 & 4.08 \\
\hline 27 & $5.16 \times 10^{19}$ & 0.62 & 63.83 & $1.28 \times 10^{-12}$ & 4.71 & 4.21 & 4.56 & 4.05 \\
\hline 28 & $5.45 \times 10^{19}$ & 0.82 & 40.67 & $9.63 \times 10^{-13}$ & 4.81 & 4.18 & 4.66 & 4.03 \\
\hline 29 & $6.86 \times 10^{19}$ & 1.44 & 20.69 & $5.85 \times 10^{-13}$ & 4.81 & 4.17 & 4.66 & 4.02 \\
\hline 30 & $8.33 \times 10^{19}$ & 1.49 & 19.68 & $5.25 \times 10^{-13}$ & 4.72 & 4.08 & 4.58 & 3.94 \\
\hline 31 & $8.94 \times 10^{19}$ & 1.25 & 24.43 & $6.49 \times 10^{-13}$ & 4.81 & 4.12 & 4.66 & 3.97 \\
\hline 32 & $9.27 \times 10^{19}$ & 1.14 & 19.50 & $5.89 \times 10^{-13}$ & 4.83 & 4.04 & 4.69 & 3.89 \\
\hline 33 & $9.87 \times 10^{19}$ & 0.95 & 33.86 & $7.92 \times 10^{-13}$ & 4.75 & 4.07 & 4.60 & 3.91 \\
\hline 34 & $1.14 \times 10^{19}$ & 0.35 & 62.50 & $1.66 \times 10^{-12}$ & 4.88 & 4.01 & 4.73 & 3.86 \\
\hline 35 & $1.31 \times 10^{19}$ & 0.96 & 19.32 & $6.23 \times 10^{-13}$ & 4.86 & 3.86 & $4.72^{2}$ & 3.71 \\
\hline
\end{tabular}


${ }^{1}$ J. del Alamo, S. Swirhun, and R. M. Swanson, Solid-State Electron. 28, 47-54 (1985).

${ }^{2}$ D. B. M. Klaassen, J. W. Slotboom, and H. C. de Graaff, Solid-State Electron. 35, 125-129 (1992).

${ }^{3}$ A. Schenk, J. Appl. Phys. 84, 3684-3695 (1998).

${ }^{4}$ D. Yan and A. Cuevas, J. Appl. Phys. 114, 044508 (2013).

${ }^{5}$ A. Cuevas, P. A. Basore, G. Giroult-Matlakowski, and C. Dubois, J. Appl. Phys. 80, 3370-3375 (1996).

${ }^{6}$ M. Rauer, M. Rüdiger, C. Schmiga, H. Strutzberg, M. Bähr, M. Glatthaar, and S. W. Glunz, J. Appl. Phys. 114, 203702 (2013).

${ }^{7}$ D. E. Kane and R. M. Swanson, in IEEE Photovoltaic Specialists Conference (IEEE, 1985), pp. 578-583.

${ }^{8}$ A. Richter, S. W. Glunz, F. Werner, J. Schmidt, and A. Cuevas, Phys. Rev. B 86, 165202 (2012).

${ }^{9}$ T. Andrew, G. Nicholas, C. Kean Fong, and K. Teng, Proceedings of the 4th International Conference on Crystalline Silicon Photovoltaics, Vol. 55 (Energy Procedia, 2014), pp. 141-148.

${ }^{10}$ A. Cuevas, R. Merchan, and J. C. Ramos, IEEE Trans. Electron Devices 40, 1181-1183 (1993).

${ }^{11}$ K. R. McIntosh and P. P. Altermatt, in 35th IEEE Photovoltaic Specialists Conference (PVSC) (IEEE, 2010), pp. 2188-2193.

${ }^{12}$ D. B. M. Klaassen, Solid-State Electron. 35, 953-959 (1992).

${ }^{13}$ K. Misiakos and D. Tsamakis, J. Appl. Phys. 74, 3293-3297 (1993).

${ }^{14}$ J. del Alamo, S. Swirhun, and R. M. Swanson, Int. Electron Devices Meet. 31, 290-293 (1985).

${ }^{15}$ P. A. Basore, IEEE Trans. Electron Devices 37, 337-343 (1990).
${ }^{16}$ A. Schenk, P. P. Altermatt, and B. Schmithusen, in 2006 International Conference on Simulation of Semiconductor Processes and Devices (IEEE, 2006), pp. 51-54.

${ }^{17}$ P. P. Altermatt, A. Schenk, B. Schmithusen, and G. Heiser, J. Appl. Phys. 100, 113715 (2006)

${ }^{18}$ J. Dziewior and J. Schmid, Appl. Phys. Lett. 31, 346-348 (1977).

${ }^{19}$ M. J. Kerr and A. Cuevas, J. Appl. Phys. 91, 2473-2480 (2002).

${ }^{20}$ P. P. Altermatt, J. Schmidt, G. Heiser, and A. G. Aberle, J. Appl. Phys. 82, 4938-4944 (1997).

${ }^{21}$ S. E. Swirhun, Y. H. Kwark, and R. M. Swanson, Int. Electron Devices Meet. 32, 24-27 (1986).

${ }^{22}$ J. Dziewior and D. Silber, Appl. Phys. Lett. 35, 170-172 (1979).

${ }^{23}$ A. Neugroschel, IEEE Electron Device Lett. 6, 425-427 (1985).

${ }^{24}$ A. B. Sproul, M. A. Green, and A. W. Stephens, J. Appl. Phys. 72, 4161-4171 (1992)

${ }^{25}$ D. D. Tang, F. F. Fang, M. Scheuermann, T. C. Chen, and G. Sai-Halasz, Int. Electron Devices Meet. 32, 20-23 (1986).

${ }^{26}$ J. Wagner and J. A. del Alamo, J. Appl. Phys. 63, 425-429 (1988).

${ }^{27}$ M. Y. Ghannam and R. P. Mertens, in 22nd European Solid State Device Research Conference, 1992, ESSDERC '92 (IEEE, 1992), pp. 691-694.

${ }^{28}$ J. W. Slotboom and H. C. de Graaff, Solid-State Electron. 19, 857-862 (1976).

${ }^{29}$ R. R. King and R. M. Swanson, IEEE Trans. Electron Devices 38, 1399-1409 (1991).

${ }^{30}$ W. P. Dumke, J. Appl. Phys. 54, 3200-3202 (1983). 